\title{
Modeling temporal variability of plasma conditions in the Io torus during the Cassini era
}

\author{
P. A. Delamere, A. Steffl, and F. Bagenal \\ Laboratory for Atmospheric and Space Physics, University of Colorado, Boulder, Colorado, USA
}

Received 10 December 2003; revised 16 June 2004; accepted 8 July 2004; published 29 October 2004.

[1] Observations of ultraviolet (UV) emissions from the major ion species $\left(\mathrm{S}^{+}, \mathrm{S}^{++}, \mathrm{S}^{++}\right.$, $\mathrm{O}^{+}, \mathrm{O}^{++}$) of the Io plasma torus made during the Cassini flyby (October 2000 to March 2001) have revealed significant time variability. Using a homogeneous model for mass and energy flow in the torus parameterized by five input variables (transport timescale, neutral source strength, ratio of oxygen to sulfur atoms in the source, fraction of superthermal electrons, and temperature of the hot electrons), we have investigated the time variability of the torus properties (density, composition, and temperature) during the Cassini era. In order to match the changes in emissions, the model suggests that a significant change in the neutral source occurred near the beginning of the observing period, decreasing from $>1.8$ tons/s to 0.7 tons/s. The changes in the neutral source appear to coincide with the declining phase of a dramatic (i.e., 2-3 order of magnitude) peak in iogenic dust emissions observed by Galileo prior to the Cassini era. INDEX TERMS: 6220 Planetology: Solar System Objects: Jupiter; 6008 Planetology: Comets and Small Bodies: Composition; 6025 Planetology: Comets and Small Bodies: Interactions with solar wind plasma and fields; 6026 Planetology: Comets and Small Bodies: Ionospheres - composition and chemistry; 6005 Planetology: Comets and Small Bodies: Atmospheres - composition and chemistry; KEYWORDS: Io, plasma torus, variability, modeling, dust, volcanoes

Citation: Delamere, P. A., A. Steffl, and F. Bagenal (2004), Modeling temporal variability of plasma conditions in the Io torus during the Cassini era, J. Geophys. Res., 109, A10216, doi:10.1029/2003JA010354.

\section{Introduction}

[2] Remote and in situ measurements of the Io plasma torus have revealed significant $(\sim 2 \mathrm{X})$ variations in density, temperature, and composition (reviewed by Thomas et al. [2004]). Long-term variations have been observed between the Voyager 1, Voyager 2, and Cassini eras, but significant short-term (i.e., few months) variations have also been observed. Brown and Bouchez [1997] report a 30\% increase in torus mass during a 6-month observing campaign in 1992 and infer a factor of 3 increase in supply to the neutral source. The cause of these variations are poorly understood, though it is possible that changes in Io's volcanic activity may be closely coupled to Io's atmosphere and the sputtering of neutral material that fuels the plasma torus [Brown and Bouchez, 1997].

[3] Recent observations of torus emissions made during the Cassini flyby of Jupiter (October 2000 to March 2001) with the Ultraviolet Imaging Spectrograph (UVIS) [Esposito et al., 2004] have provided a unique opportunity to investigate torus short-term variations during a 6-month period in detail. The UVIS instrument made measurements of the emissions from all of the major ionized species, and the analysis of these emissions provides estimates of density, composition, and temperatures in the region between 6 and

Copyright 2004 by the American Geophysical Union. 0148-0227/04/2003JA010354\$09.00
$7.5 R_{J}$ where the majority of the mass and energy in the torus is found [Steffl et al., 2004a, 2004b].

[4] The dramatic variations seen in the torus emissions during the Cassini era suggest that the torus conditions were rapidly changing (Figure 1). Interestingly, observations of jovian dust streams made during Galileo's G28 orbit show a $2-3$ order of magnitude increase in dust emission rate from Io [Krüger et al., 2003] prior to the Cassini observing period (see Figure 2). The enhanced dust emissions began in July 2000 , peaked in September 2000 at $\sim 10^{2} \mathrm{~kg} / \mathrm{s}$, and returned to a nominal background level of $\sim 10^{-1} \mathrm{~kg} / \mathrm{s}$ by December 2000. While the relation between dust emissions and the production of neutral gas is not known, Krüger et al. [2003] argues that the dust streams serve as a proxy for Io's volcanic plume activity. Between February 2000, and December 2000, large-area surface changes associated with the Tvashtar volcano occurred and sightings of the Tvashtar plume were made in December 2000. We postulate that enhanced neutral gas production is a likely byproduct of the volcanic plume activity that generated the dust emission "spike" observed before and during the Cassini era. In addition, we note that the Cassini event may be similar to the 1992 outburst on Io reported by Brown and Bouchez [1997].

[5] Numerous models of mass and energy flow through the torus suggest that a neutral source of $\sim 1$ ton/s is sufficient to produce the dense $\left(\sim 2000 \mathrm{~cm}^{-3}\right)$ sulfur- and oxygen-dominated torus [Shemansky, 1988; Barbosa, 1994; Schreier et al., 1998; Lichtenberg and Thomas, 2001; Smyth 


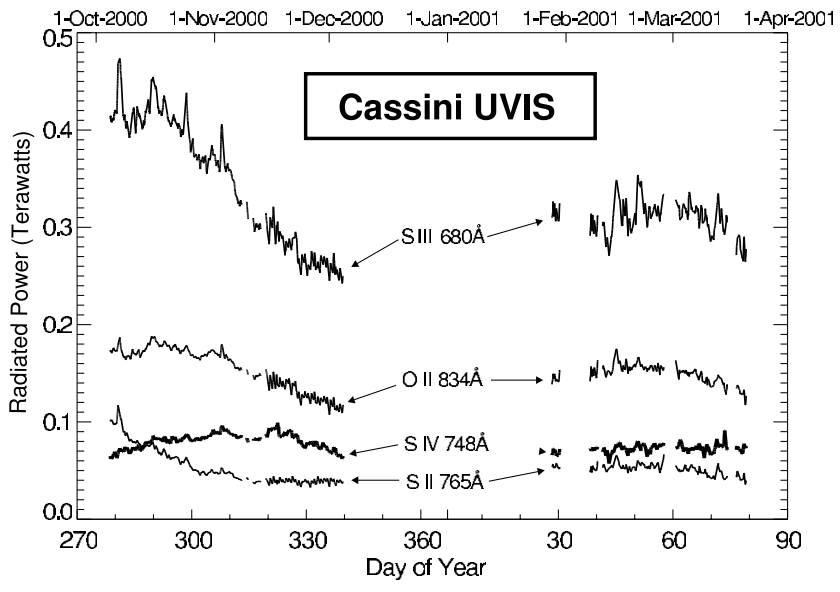

Figure 1. Variations in power emitted in the brightest lines of the four major ion species from October 2000 through March 2001 (Cassini UVIS) [Steffl et al., 2004b].

and Marconi, 2003; Delamere and Bagenal, 2003]. Energy is added to the torus through the pickup of ionized neutral material and through a nonthermal component of the electron velocity distribution. A significant energy sink $(\sim 50 \%)$ is ultraviolet (UV) and optical radiation.

[6] We have adopted a homogeneous, time-dependent model for studying the flow of mass and energy in the torus during the Cassini era [Delamere and Bagenal, 2003]. While density, composition, and temperature are known to vary spatially, the homogeneous model is a reasonable approximation for the region between 6 and $7.5 R_{J}$ where composition varies weakly with radius [Steffl et al., 2004b]. We also assume that a significant source of mass is provided by Io's extended neutral clouds, as implied by the Galileo J0 flyby [Bagenal et al., 1997; Saur et al., 2003] and that the bulk of the plasma is picked up approximately at corotation velocity. The goal of this study is to model the observed torus emissions starting with the simplest model and adding complexity as warranted to fit the data. Specifically, we apply a steady state model to broadly constrain the model input parameters for times where the torus is likely to be in a quasi-equilibrium configuration (i.e., beginning and end of the observed declining emissions) and then consider timedependent models to understand the primary mechanisms responsible for the observed variability.

\section{Cassini Dataset}

[7] During the Cassini spacecraft's Jupiter flyby (October 2000 to March 2001), the UVIS instrument produced an extensive data set of spectrally dispersed images of the Io plasma torus. The UVIS instrument consists of two independent, coaligned spectrographs (EUV 561-1181 A; FUV $1115-1913 \AA$ ) having a point-source spectral resolution of 3 A FWHM [McClintock et al., 1993; Esposito et al., 1998]. The major ion species present in the torus all have spectral features in the wavelength range covered by the EUV channel. The field of view of the EUV channel of the instrument was such that for the first and last 2 months of the Jupiter encounter, the entire torus could be observed simultaneously. During the approach phase of the encoun- ter, the total EUV power radiated by the torus decreased by $25 \%$ [Steffl et al., 2004b]. The total power radiated from the torus in four representative spectral features is shown in Figure 1. UVIS continued to make observations of the Io torus during the months of December 2000 and January 2001 , but the spacecraft's proximity to Jupiter precluded simultaneous measurement of the total radiated EUV power. When total power measurements resumed after closest approach, the torus EUV luminosity remained relatively constant.

[8] The spectral analysis method described by Steffl et al. [2004a] was used to derive the ion composition and electron temperature of the torus plasma at the ansa. This model assumes a uniform torus along the line-of-sight and uses the CHIANTI database [Dere et al., 1997; Young et al., 2003] to determine radiative emission rates. The assumption of a uniform column through the torus does not significantly affect the results for radial distances greater than $6 R_{J}$. The derived torus composition, averaged over system III longitude, on 1 October, 14 Novemeber, and 14 January is presented in Table 1. Spectra from the October and November dates can be found in the work of Steffl et al. [2004b]; spectra from the January date can be found in the work of Steffl et al. [2004a].

[9] Historically, it has been difficult to determine the relative abundance of $\mathrm{O}$ II and $\mathrm{O}$ III in the Io torus [McGrath et al., 1993, and references therein] The observational setup of UVIS on 14 January 2001 (i.e., the long axis of the UVIS entrance slit oriented approximately parallel to Jupiter's rotation axis with the field of view being scanned radially inward from 10 to $4 R_{J}$ ) allowed the inclusion of the FUV channel in the analysis of the spectra. The observed brightness of the O III lines at $1661 \AA$ and

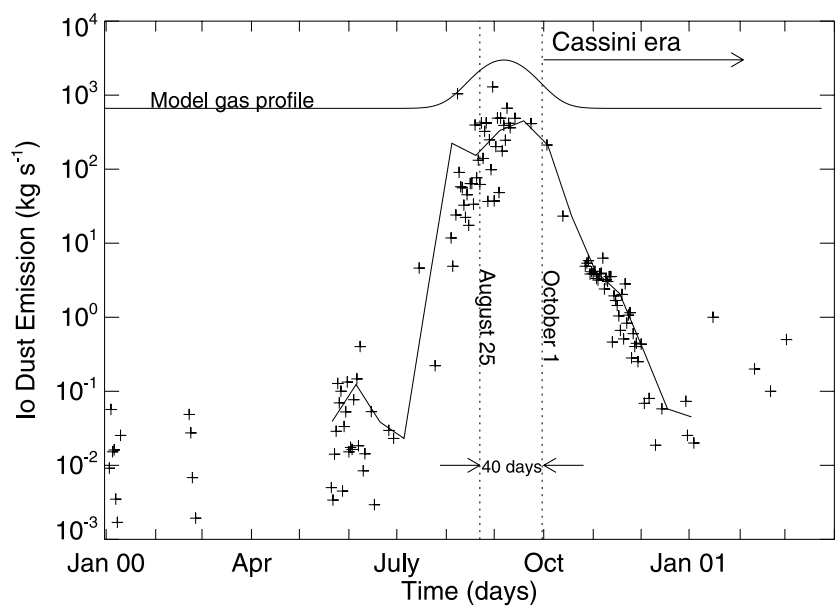

Figure 2. Dust emission rates from Krüger et al. [2003]. The data symbols are 1 day averages of the dust emissions, and the solid line is a 15 day average. The Cassini observations of the torus began in October 2000, indicated by the dashed line. The dotted lines mark the 40-day interval prior to the Cassini observations and represent the torus equilibration time. The dust emissions do not vary drastically during this 40-day window suggesting that the torus may have reached a quasi-steady state for the first Cassini observations. The model neutral gas production rate is shown for reference at the top. 
Table 1. Comparison of Average Torus Conditions as Observed by Cassini During a 3 Month Observing Period With Best Fit Parameters From the Homogeneous Model

1 Oct 200014 Nov 200014 Jan 2001

\begin{tabular}{lccc}
\hline \multicolumn{4}{c}{ Cassini Observations $^{\mathrm{a}}$} \\
$n_{\mathrm{S}^{+}} / n_{e}, \%$ & 10 & 4.7 & 6.0 \\
$n_{\mathrm{S}^{++}} / n_{e}, \%$ & 22 & 20 & 21 \\
$n_{\mathrm{S}^{++}} / n_{e}, \%$ & 2.2 & 4.5 & 3.7 \\
$n_{\mathrm{O}^{+}} / n_{e}, \%$ & 26 & 28 & 27 \\
$n_{\mathrm{S}^{++}} / n_{e}, \%$ & 1.7 & 1.8 & 1.7 \\
Total EUV power, $10^{12} \mathrm{~W}$ & $2.0 \pm 0.2$ & $1.5 \pm 0.2$ & $1.5^{\mathrm{b}}$ \\
$\quad$ Volumetric EUV $^{\mathrm{b}}$ & $0.40 \pm 0.04$ & $0.30 \pm 0.04$ & $0.30^{\mathrm{b}}$ \\
$\quad$ powerc \\
\end{tabular}

Best Fit Model Parameters

\begin{tabular}{lcc}
\multicolumn{3}{c}{ Best Fit Model Parameters } \\
Neutral source $\mathrm{O} / \mathrm{S}$ ratio & 1.7 & 1.9 \\
Neutral source, $10^{-4} \mathrm{~cm}^{-3} \mathrm{~s}^{-1}$ & 17.2 & 6.4 \\
Neutral source, ${ }^{\mathrm{d}}$ tons $/ \mathrm{s}^{-1}$ & 1.8 & 0.7 \\
Transport time, $\tau$, days & 27 & 64 \\
Hot electron fraction, $f_{e h}, \%$ & 0.23 & 0.25 \\
Hot electron temperature, $T_{e h}, \mathrm{eV}$ & 80 & 46
\end{tabular}

${ }^{a}$ Mixing ratios assume $[\mathrm{H} \mathrm{II}]=.1 *[\mathrm{e}]$. Mixing ratio uncertainty $\sim 10 \%$ [Steffl et al., 2004a].

${ }^{b}$ We assume that the January to March total EUV power is similar to the total EUV power on 14 November based on the similarity of the line emissions.

${ }^{\mathrm{c}}$ Assumes an equivalent torus volume of $3.1 \times 31 \mathrm{~cm}^{3}$.

${ }^{\mathrm{d}}$ Assumes $m_{i}=20 * m_{p}$.

$1666 \AA$, a wavelength region covered by the FUV channel, places a firm upper limit the amount of O III in the torus. Since it was not possible to use the FUV channel in the analysis of the October and November data, the amount of O III present in the torus could not be directly determined. We therefore assume that the upper limit on the O III/O II ratio was the same for October and November of 2000 as it was on 14 January 2001.

\section{Model of Torus Chemistry and Emissions}

[10] We have developed a homogeneous, time-dependent model of torus physical chemistry for the purpose of investigating the sensitivity of torus composition to the following parameters: neutral source rate $\left(\mathcal{S}_{n}\right), \mathrm{O} / \mathrm{S}$ source ratio $(\mathrm{O} / \mathrm{S})$, convective transport loss $(\tau)$, hot electron fraction $\left(f_{e h}\right)$, and hot electron temperature $\left(T_{e h}\right)$. A detailed description of the model is provided by Delamere and Bagenal [2003]. The model is based largely upon several earlier models [i.e., Shemansky, 1988; Barbosa, 1994; Schreier et al., 1998; Lichtenberg and Thomas, 2001] but uses the latest CHIANTI atomic physics database for computing radiative loss [Dere et al., 1997].

[11] The model calculates the time rate of change of mass and energy for both ions and the core electrons based on the determination of mass and energy sources and losses until a steady state solution is reached. The primary sources of mass and energy are electron impact ionization and charge exchange reactions involving neutral gas. Charge exchange reactions determine the allocation of energy among the ion species and their respective ionization states. Charge exchange reactions involving neutrals also contribute significantly to the energy budget due to the pickup energy of plasma into the corotating plasma torus (see Table 1 of
Delamere and Bagenal [2003] for reactions). The velocity distribution for each species is approximated as Maxwellian. The electrons have a nonthermal component and a $\kappa$-distribution is known to best match torus observations [Meyer-Vernet et al., 1995]. We approximate this nonthermal distribution with two Maxwellians for the core and hot populations. The hot electrons, through coulomb coupling with the core electrons, provide significant energy input into the torus $(\sim 20-60 \%)$. Major losses of mass include radial transport and fast neutral escape due to charge exchange reactions with thermalized ions $\left(T_{i}=70-100 \mathrm{eV}\right)$. Radiation in the UV and optical is the major energy sink as roughly $50 \%$ of the input energy is transferred from the ions to the electrons via coulomb coupling. The combination of these sources and sinks of mass and energy lead to an equilibration timescale of roughly 40 days.

[12] The homogeneous approximation simplifies, in particular, the convective transport process which is known to be spatially variable. However, if one considers the torus between $6 R_{J}$ and $7.5 R_{J}$ where $\sim 80 \%$ of the mass and energy is found, then the homogeneous model is a reason-
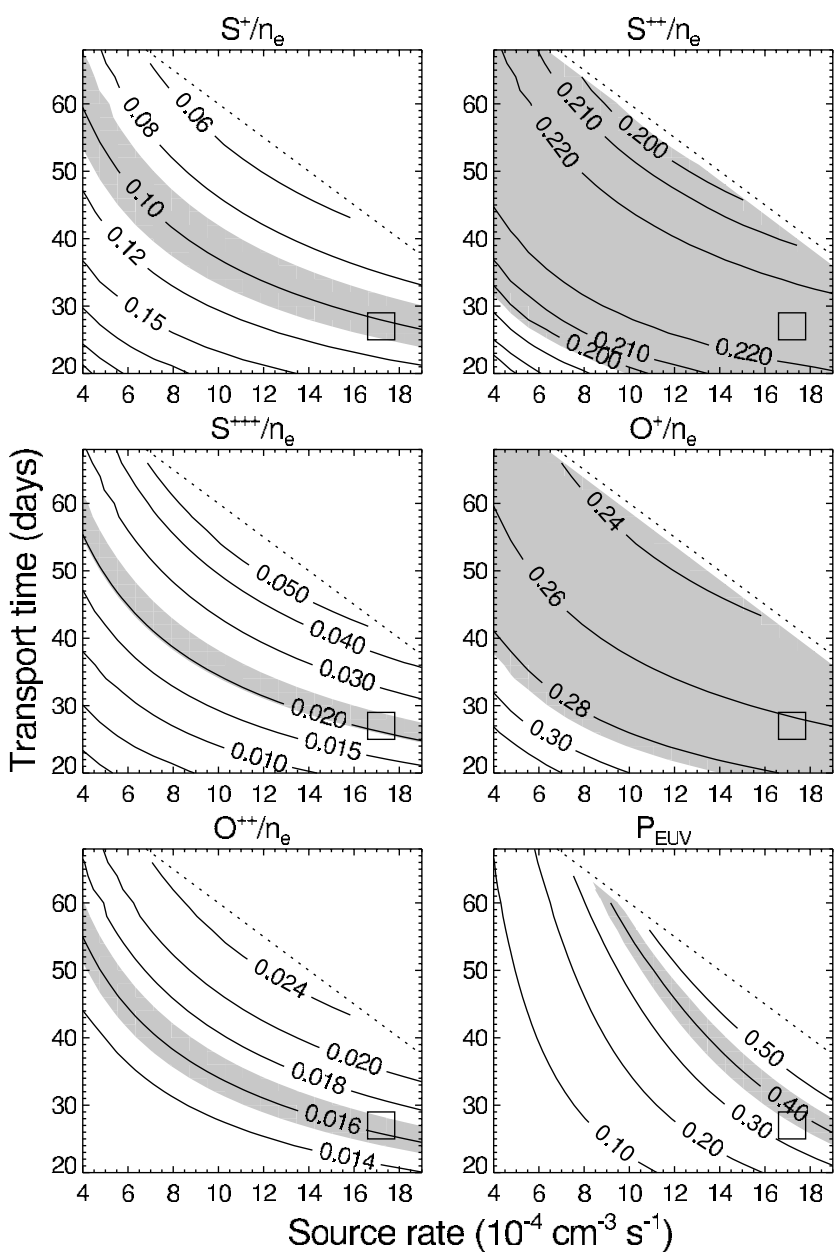

Figure 3. Model results for October plasma conditions showing mixing ratios for the five major ion species and the total EUV luminosity as functions of transport time and neutral source rate. The best fit is shown by the square for $f_{e h}=0.23 \%, T_{e h}=80 \mathrm{eV}$, and $\mathrm{O} / \mathrm{S}=1.7$. The shaded regions indicate uncertainties in the Cassini UVIS analysis. 


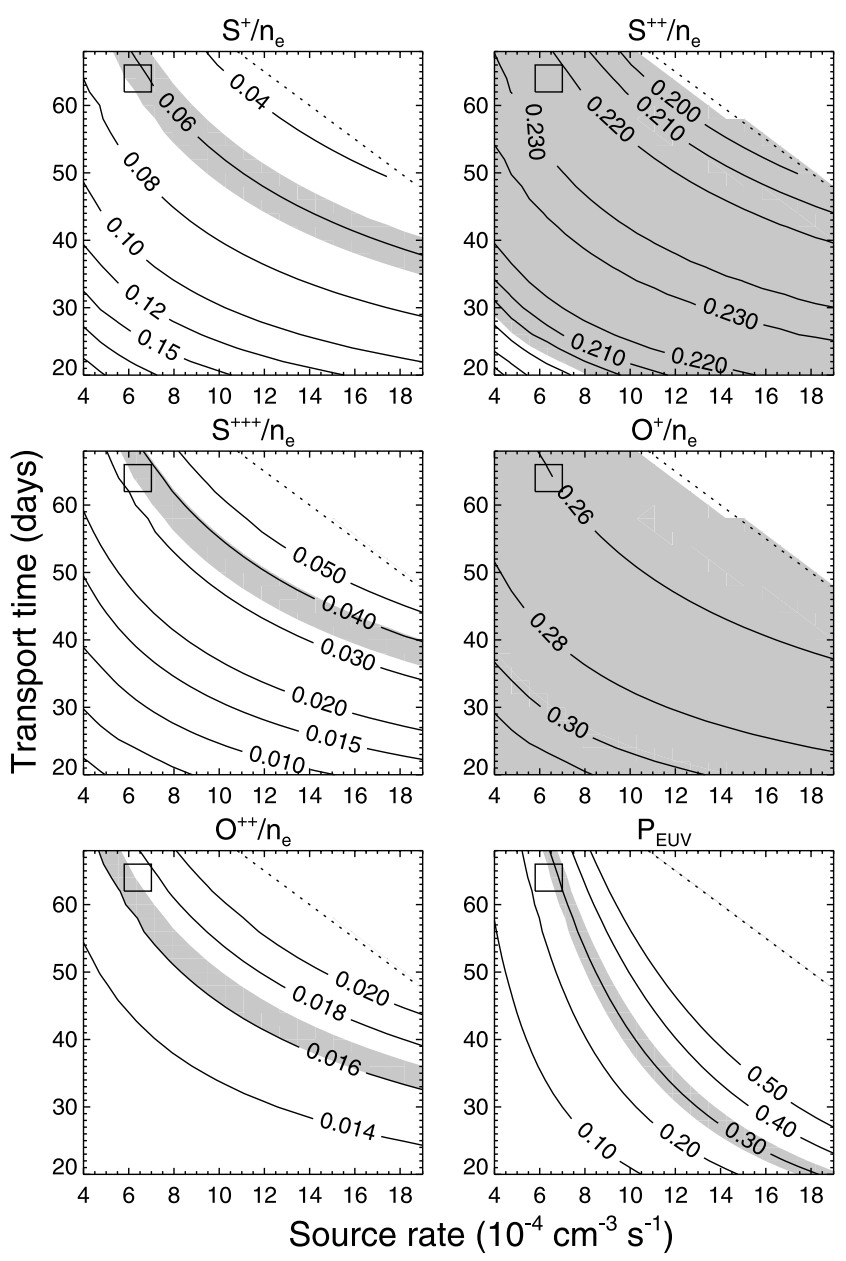

Figure 4. Model results for January plasma conditions showing mixing ratios for the five major ion species and the total EUV luminosity as functions of transport time and neutral source rate. The best fit is shown by the square for $f_{e h}=0.25 \%, T_{e h}=46 \mathrm{eV}$, and $\mathrm{O} / \mathrm{S}=1.9$. The shaded regions indicate uncertainties in the Cassini UVIS analysis.

able approximation since negligible mass is added to the volume at Io's location $\left(\sim 6 R_{J}\right)$ boundary through transport processes. For the purpose of converting between volumetric quantities and volume-integrated quantities, we use an equivalent torus volume of $3.1 \times 10^{31} \mathrm{~cm}^{3}$, or $85 R_{J}^{3}$, which is based on fits of volumetric model output to the volumeintegrated Cassini results for the total EUV luminosity. This volume also happens to be the volume of a geometrical torus bounded by 6.0 and $7.6 R_{J}$ which is nearly identical to the region of the torus where we consider the homogeneous approximation to be valid (i.e., where composition is roughly constant [Steffl et al., 2004a]).

\subsection{Steady-State Models}

[13] Our initial approach is to model the declining emissions at the beginning (1 October) and end (14 January) as steady state conditions. If the hypothesized neutral source enhancement was abruptly turned on at least 40 days prior to the Cassini observing window, then the torus can be considered in a quasi-steady state by 1 October 2000 . The dotted vertical lines in Figure 2 indicate the 40-day period prior to the beginning of the Cassini observations on 1 October. If we assume that the dust emissions are coupled to iogenic neutral gas production, then it is possible that a quasi-steady state was reached by 1 October given the relative flatness of the dust emission rates. However, the evolution of the torus after 1 October is likely a nonequilibrium state and must be considered with a time-dependent model. Based on the flatness of the line emission profiles, the steady state assumption is likely valid again for January to March, 2001. Using the downhill simplex method of Nelder and Mead [1965], steady state solutions were found for the torus conditions on 1 October 2000 and 14 January 2001.

\subsection{Steady State Solutions}

[14] A comprehensive five-dimensional parameter space search using the downhill simplex method of Nelder and Mead [1965] isolated two primary regions where the model results match the observed Cassini plasma conditions. The observed October plasma conditions are matched by a region of model parameter space that is distinctly different from the January model fits. Best fit model parameters for the October and January cases are given in Table 1. To further justify the steady state treatment of the October and January cases, we note that for intermediate cases (e.g., 14 November 2000) the steady state model fits are poor; that is, we cannot simultaneously match the model results to the six observables discussed below. Figures 3 and 4 show model results for October and January illustrating the sensitivity of torus composition to transport time, $\tau$, and neutral source rate, $\mathcal{S}_{n}$, for a fixed hot electron fraction, hot electron temperature, and neutral $\mathrm{O} / \mathrm{S}$ source ratios (see Delamere and Bagenal [2003] for further discussion of model sensitivity to parameters). The six panels, ordered from left to right and top to bottom, show the sulfur ion mixing ratios (i.e., $n_{\mathrm{S}^{n}} / n_{e}$ ), the oxygen ion mixing ratios, and finally the EUV luminosity. The shaded regions show the measured Cassini values $( \pm 10 \%)$ and the volume-integrated power radiated (texperimental uncertainty) converted to a volumetric emission rate. The shaded regions also illustrate the relative sensitivity of the ion mixing ratios to variations in $\mathcal{S}_{n}$ and $\tau$ with the major ion species (i.e., $\mathrm{O}^{+}$ and $\mathrm{S}^{++}$) being relatively insensitive and the minor species (i.e., $\mathrm{O}^{++}, \mathrm{S}^{+}$and $\mathrm{S}^{+++}$) being highly sensitive. As a result, the minor species largely determine the model parameter fits. The boxed regions show our best fits and illustrate an unquantified region of parameter space that encompasses uncertainties in the atomic data and errors due to the simplifying assumptions of the homogeneous model. It is important to note that the radiation contours contain an orthogonal component to the mixing ratio contours and thus $P_{E U V}$ (along with the torus volume) constrains the model fit. Without the $P_{E U V}$ constraint, the mixing ratios alone would span a large region of parameter space from small source rate/large transport time to large source rate/small transport time.

[15] Figure 5 shows the sensitivity of model results to variations in the remaining three parameters: $\mathrm{O} / \mathrm{S}, f_{e h}$, and $T_{e h}$. The three panels are the sulfur mixing ratios, the oxygen mixing ratios, and the EUV power emission rate. The shaded regions are the uncertainty range for the January Cassini results. The seven trial cases are shown in the 

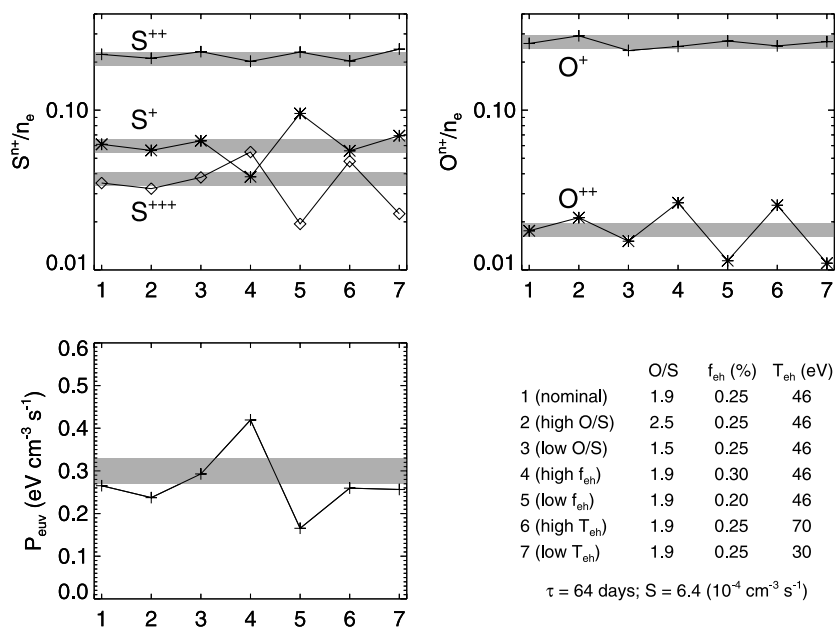

$\begin{array}{llll} & \mathrm{O} / \mathrm{S} & \mathrm{f}_{\text {en }}(\%) & \mathrm{T}_{\text {eh }}(\mathrm{eV}) \\ 1 \text { (nominal) } & 1.9 & 0.25 & 46 \\ 2 \text { (high O/S) } & 2.5 & 0.25 & 46 \\ 3(\text { low O/S) } & 1.5 & 0.25 & 46 \\ 4\left(\text { high } \mathrm{f}_{\text {en }}\right) & 1.9 & 0.30 & 46 \\ 5\left(\text { low } \mathrm{f}_{\text {en }}\right) & 1.9 & 0.20 & 46 \\ 6\left(\text { high } \mathrm{T}_{\text {eh }}\right) & 1.9 & 0.25 & 70 \\ 7\left(\text { low } \mathrm{T}_{\text {en }}\right) & 1.9 & 0.25 & 30 \\ \quad \tau=64 \text { days; } \mathrm{S}=6.4\left(10^{-4} \mathrm{~cm}^{-3} \mathrm{~s}^{-1}\right)\end{array}$

Figure 5. Sensitivity of hot electron fraction, hot electron temperature, $\mathrm{O} / \mathrm{S}$ neutral source ratio for fixed transport time $(\tau=64$ days $)$, and neutral source rate $\left(S_{n}=6.4 \times\right.$ $\left.10^{-4} \mathrm{~cm}^{-3} \mathrm{~s}^{-1}\right)$. The parameters for seven cases are shown. The shaded regions show the uncertainty range for the 14 January Cassini observations.

bottom right hand corner. Case one is the best fit model result for January and the remaining cases show variations in composition for increasing and decreasing $\mathrm{O} / \mathrm{S}, f_{e h}$ and $T_{e h}$, respectively. Composition is relatively insensitive to variations in the $\mathrm{O} / \mathrm{S}$ ratio, suggesting that differences between the October and January best model fits should not be considered significant. Composition is more sensitive to variations in the nonthermal electron parameters $\left(f_{e h}\right.$ and $\left.T_{e h}\right)$, and we conclude that differences between the October and January cases are significant.

[16] The primary conclusion of this parameter search is that the torus conditions changed substantially between October and January. For October the best fit required a neutral source of roughly $17 \times 10^{-4} \mathrm{~cm}^{-3} \mathrm{~s}^{-1}(\sim 1.8$ tons/s) with an $\mathrm{O} / \mathrm{S}$ source ratio of 1.7 . The January fit required a source rate of roughly $3 \mathrm{X}$ smaller, or $6.4 \times 10^{-4} \mathrm{~cm}^{-3} \mathrm{~s}^{-1}$ $(\sim 0.7$ tons $/ \mathrm{s})$, and an $\mathrm{O} / \mathrm{S}$ source ratio of 1.9 .

\subsection{Time-Dependent Model}

[17] The steady state approximation seems reasonable for the 1 October and 14 January cases, but to model the intermediate period we need a time-dependent model. Our computational method can be used for time-dependent modeling, though such solutions are not unique given the independent treatment of the five input parameters. There are a few obvious interdependencies that can be used to loosely constrain time-dependent variation of the input parameters. For example, one expects an inverse relationship between the neutral source rate and transport (i.e., increasing the neutral source rate increases the torus density gradient which drives more rapid radial transport via interchange motion [Southwood and Kivelson, 1989; Brown and Bouchez, 1997; Pontius et al., 1998]).

[18] We are motivated to consider significant changes in the neutral source rate based on the enhanced dust emissions. Our highly simplified time-dependent model uses the best fit model input parameters for the January (final) steady state solution and assumes a Gaussian perturbation to the neutral source rate of the form $\left.\delta N=\alpha N_{o} e^{-\left(t-t_{o}\right.}\right)^{2} / \delta t^{2}$ where $t_{o}=250$ days (5 September 2000), $N_{o}$ is the background neutral source rate (January best fit), $\delta t$ is the width of the Gaussian, and $\alpha$ scales the amplitude of the perturbation. Figure 2 shows the neutral perturbation for $\alpha=3.5$ and $\delta t=$ 22.5 days. We assume an inverse relation between transport and the neutral source. In this preliminary model we fix the hot electron fraction $\left(f_{e h}=0.25 \%\right)$ and temperature $\left(T_{e h}=46 \mathrm{eV}\right)$ and the neutral source ratio $(\mathrm{O} / \mathrm{S}=1.9)$ at the 14 January steady state model values.

\subsection{Time-Dependent Model Results}

[19] Figures 6-8 show the time-dependent model results for neutral source amplitude variations with $\alpha=2.0,3.5$, and 5.0, respectively. The Gaussian width was chosen to be 22.5 days. The top panel shows the ion mixing ratios and the bottom panel shows the power radiated for the observed line emissions shown in Figure 1. The symbols are from the Cassini UVIS analysis of [Steffl et al., 2004a, 2004b]. Observational uncertainties are indicated $( \pm 10 \%)$ for the first and last data points and the arrows indicate that the $\mathrm{O}^{++}$ values represent a firm upper limit.

[20] Qualitatively, the simple time-dependent model shows remarkable agreement with the data. The dramatic decrease/increase of the $\mathrm{S}^{+} / \mathrm{S}^{+++}$mixing ratios is reproduced, while the $\mathrm{O}^{+}, \mathrm{O}^{++}$, and $\mathrm{S}^{++}$mixing ratios are all consistent within experimental uncertainty. Note that the model is relaxing to the 14 January steady state solution, so differences between the observations and the model results in November reflect, perhaps, additional variations in the neutral source that are not included in the model. The

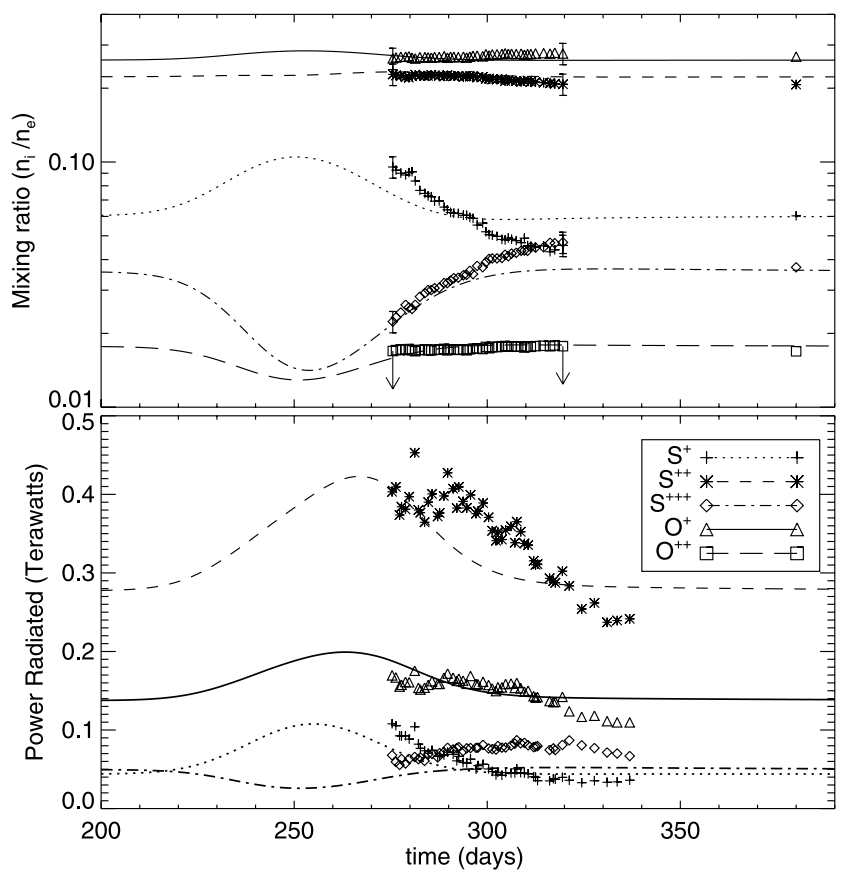

Figure 6. Time variation of the brightest lines of the 4 major ion species (Figure 1) for a neutral source perturbation described by $\alpha=2.0$ and $\delta t=22.5$ days. The symbols are samples of the UVIS measurements and the lines are model output. 


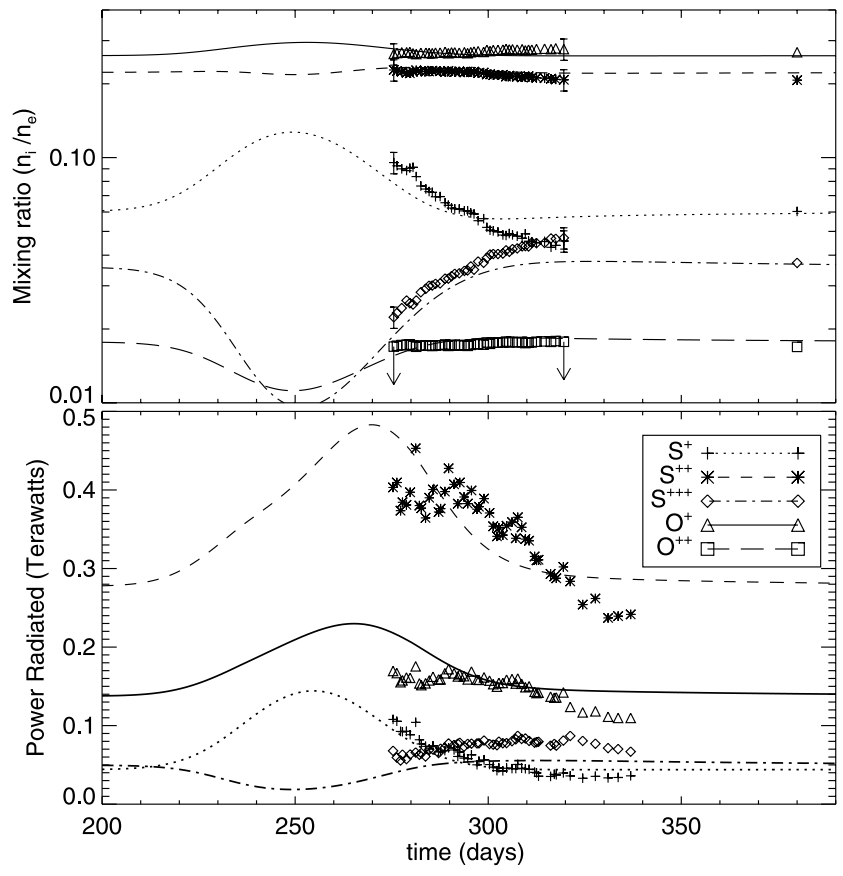

Figure 7. Time variation of the brightest lines of the four major ion species (Figure 1) for a neutral source perturbation described by $\alpha=3.5$ and $\delta t=22.5$ days. The symbols are samples of the UVIS measurements and the lines are model output.

modeled $\mathrm{S}^{+}$and $\mathrm{S}^{++}$line emissions are in reasonable agreement with the observations. Initially, the modeled $\mathrm{O}^{+}$ $834 \AA$ line emission is $\sim 50 \%$ brighter than observed and the $\mathrm{S}^{+++} 748 \AA$ line emission is consistently lower than observed. However, differences in the modeled emissions with the Cassini observations should not be interpreted too rigorously. The emission rates are dependent on ion density and on electron density and temperature. The variation in the $\mathrm{S}^{+}$and $\mathrm{S}^{+++}$line emissions are driven largely by the changes in the respective ion densities, while variations in the $\mathrm{O}^{+}$and $\mathrm{S}^{++}$emission rates mostly depend on the variations in electron density and temperature. Given that the physical torus is spatially inhomogeneous, small changes (e.g., 20\%) in the electron density and temperature can account for the differences between the model results and the Cassini observations. Perhaps the best fit is for the case shown in Figure 7 for $\alpha=3.5$, or a peak neutral source rate of 3.0 tons/s (compared to our nominal January source rate of 0.64 tons $/ \mathrm{s}$ ). The 40 -day average neutral source prior to 1 October for these cases is 2.4 tons/s, somewhat larger than the steady state solution for 1 October of 1.8 tons $/ \mathrm{s}$.

[21] Figure 9 compares the model results for neutral density, ion/electron density, ion/electron temperature, mixing ratios, and power radiated for the best fit case shown in Figure $7(\alpha=3.5)$. The neutral density enhancements are obviously due to the increased neutral source rate. The density of the singly ionized oxygen and sulfur are primarily the result of electron impact ionization of $\mathrm{O}$ and $\mathrm{S}$ and increase, as expected, with the increased neutral density. The ion temperatures correspondingly increase due to the abundance of new pickup ions. The dominant loss processes for $\mathrm{S}^{+++}$and $\mathrm{O}^{++}$are likely charge exchange reactions involving $\mathrm{O}$ and $\mathrm{S}$ with relatively large reaction rates of $\sim 1.9 \times 10^{-8} \mathrm{~cm}^{3} \mathrm{~s}^{-1}$ and $\sim 1.6 \times 10^{-8} \mathrm{~cm}^{3} \mathrm{~s}^{-1}$, respectively. Thus the increased neutral density leads to a decrease in the density of these higher ionized species. The electron densities and temperature do not vary significantly (i.e., $\sim 10 \%$ and $\sim 20 \%$, respectively) but nevertheless strongly affect the emission rates. The increase in the electron temperature follows from coupling to a hotter ion population, while the decrease in electron density follows from the combination of transport losses and the relative decrease in the abundance of the higher ionization states. These variations in electron density and temperature together yield the observed $25 \%$ variation in the total EUV power radiated.

[22] Table 2 compares the modeled mass and energy flow for the case of 5 September 2000 (peak neutral production) with the 14 January 2001 steady state solution. The mass flow is basically unchanged by the enhanced neutral source (see mass sources and losses). The $\mathrm{S}$ and $\mathrm{O}$ input are fixed by the $\mathrm{O} / \mathrm{S}$ ratio and transport and fast neutral losses are roughly equal for both cases. The total power radiated only increases by roughly $20-25 \%$, as most of the additional pickup power is diverted into transport and fast neutral loss. For the October case shown in Table $2(\alpha=3.5)$, transport, fast neutral, and radiative losses are comparable. The hot electron input is nearly the same for both cases as expected for the same hot electron fraction, and the hot electron temperature does not strongly influence the energy flow. The increase in the core electron temperature seen in Figure 9 is largely due to the thermal coupling with the ions. These results hinge on the the assumption that $\tau \propto 1 / \mathcal{S}_{n}$, but based on the qualitative agreement between the model results and

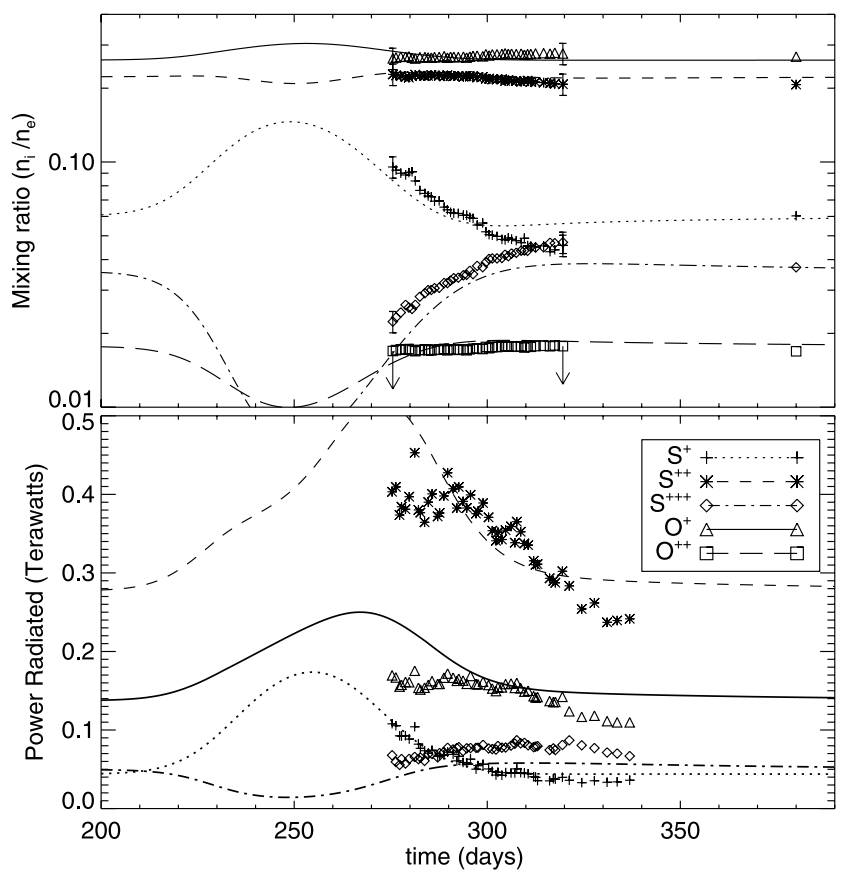

Figure 8. Time variation of the brightest lines of the four major ion species (Figure 1) for a neutral source perturbation described by $\alpha=5.0$ and $\delta t=22.5$ days. The symbols are samples of the UVIS measurements and the lines are model output. 

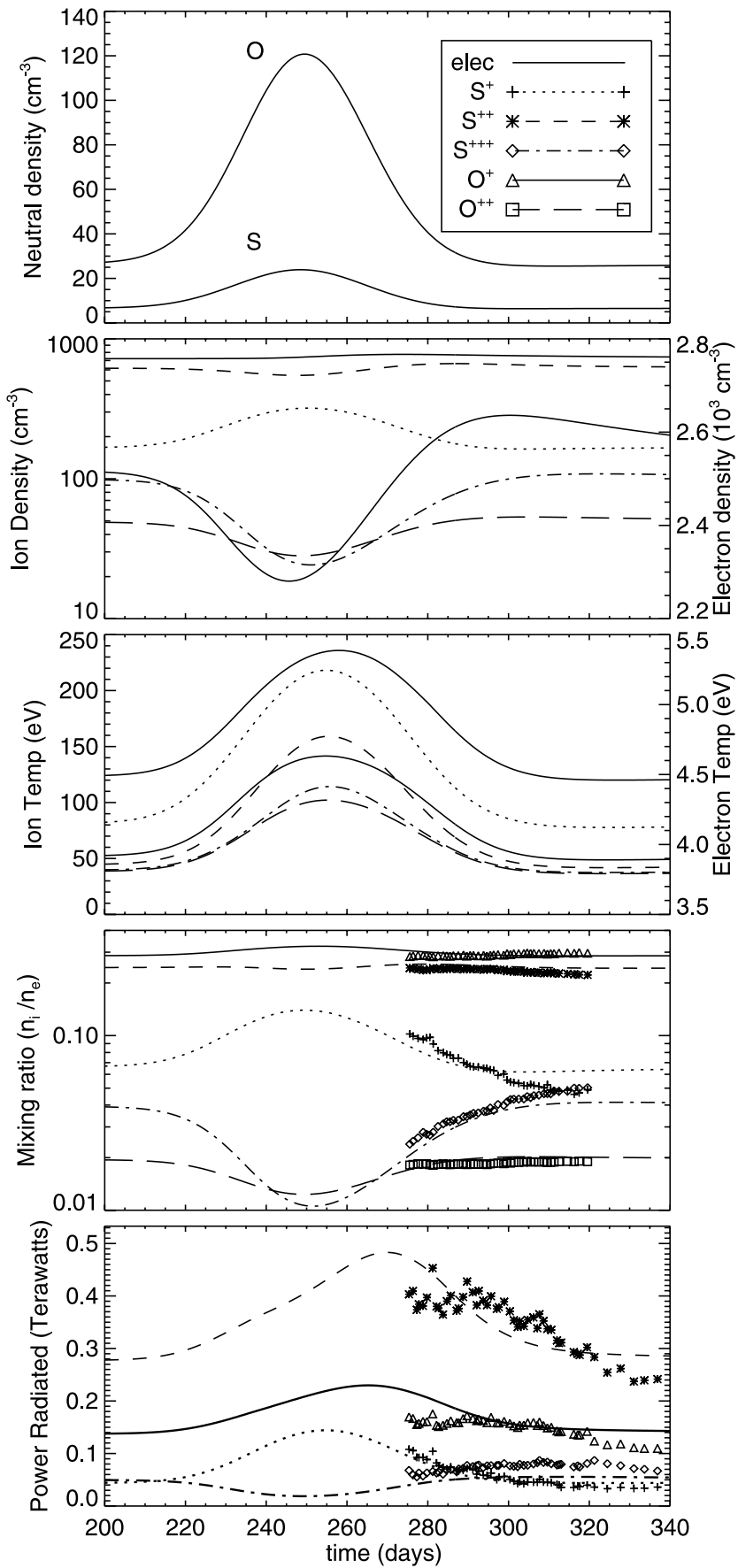

Figure 9. Model output for the best fit case $(\alpha=3.5)$. The panels show neutral density, ion/electron density, ion/ electron temperature, ion mixing ratios, and power radiated for the brightest lines fo the four major ion species.

the Cassini observations this seems to be a reasonable assumption.

\section{Discussion}

[23] Initial studies of torus variability [Delamere and Bagenal, 2003] showed considerable differences in the torus properties between the Voyager 1, Voyager 2, and Cassini eras. Delamere and Bagenal [2003] concluded that volu- metric neutral source rates varied from 0.5 tons/s to 1.3 tons/s. While it might not be surprising to observe such variations over a long period of time (i.e., years), it is interesting to observe strong variations in neutral source over a much shorter time (i.e., months). Figure 1 illustrates what may be interpreted as a sudden change in the neutral source near the beginning of the observation period. During the roughly 6 week interval from 1 October to 14 November, the brightest $\mathrm{S}^{++}$line steadily decreased. The model results yield similar torus properties for the November and January cases, suggesting a steady neutral source rate of $\sim 0.7$ tons/s. For the remainder of the observing period the torus properties are roughly constant. In addition, the response of the torus between October and November to a rapid change in the neutral source is consistent with the $\sim 40$ day timescale for many of the dominant physical and chemical processes that drive the torus. Figures 6-8 illustrate this point, showing the time-dependent model results for radiated power of the four major ion species and the ionmixing ratios for the case where the neutral source rate is varied over a timescale consistent with the observed Io dust emissions. The qualitative agreement between the observed radiated power and the time-dependent model results suggests that indeed a significant change occurred. Without observations of torus composition and power emitted prior to 1 October, it is impossible to uniquely constrain the neutral source; however, the general agreement between our steady state and simple time-dependent models with the

Table 2. Comparison of Energy Throughput for Peak Neutral Production (5 September 2000) and Nominal Steady-State (14 January 2001)

\begin{tabular}{|c|c|c|}
\hline & 5 Sept 2000 & 14 Jan 2001 \\
\hline \multicolumn{3}{|c|}{ Input Parameters } \\
\hline Neutral source, $c a l S_{n}, 10^{-4} \mathrm{~cm}^{-3} \mathrm{~s}^{-1}$ & 29 & 6.4 \\
\hline Transport time, $\tau$, days & 14 & 64 \\
\hline Neutral source $\mathrm{O} / \mathrm{S}$ ratio & 1.9 & 1.9 \\
\hline Hot electron fraction, $f_{e h}, \%$ & 0.25 & 0.25 \\
\hline Hot electron temperature, $T_{e h}, \mathrm{eV}$ & 90 & 46 \\
\hline \multicolumn{3}{|c|}{ Mass Sources, \% } \\
\hline S pickup & 34 & 34 \\
\hline O pickup & 66 & 66 \\
\hline \multicolumn{3}{|c|}{ Mass Losses, \% } \\
\hline Fast neutrals & 55 & 53 \\
\hline Transport & 45 & 47 \\
\hline \multicolumn{3}{|c|}{ Energy Flow, $\mathrm{eV} \mathrm{cm}^{-3} \mathrm{~s}^{-1}$} \\
\hline Total energy & 1.35 & 0.59 \\
\hline \multicolumn{3}{|c|}{ Energy Sources, $\mathrm{eV} \mathrm{cm}^{-3} \mathrm{~s}^{-1}, \%$} \\
\hline $\mathrm{S}$ ionization & $0.34(24)$ & $0.07(11)$ \\
\hline S charge exchange & $0.21(16)$ & $0.05(8)$ \\
\hline $\mathrm{O}$ ionization & $0.13(9)$ & $0.02(3)$ \\
\hline $\mathrm{O}$ charge exchange & $0.39(29)$ & $0.10(17)$ \\
\hline Hot/cold electron thermal coupling & $0.28(21)$ & $0.36(61)$ \\
\hline \multicolumn{3}{|c|}{ Internal Thermal Coupling, $\mathrm{eV} \mathrm{cm}^{-3} \mathrm{~s}^{-1}, \%$} \\
\hline Ion-electron thermal coupling & $0.38(28)$ & $0.19(32)$ \\
\hline \multicolumn{3}{|c|}{ Energy Losses, $\mathrm{eV} \mathrm{cm}^{-3} \mathrm{~s}^{-1}, \%$} \\
\hline Fast neutrals & $0.34(26)$ & $0.03(4)$ \\
\hline Transport & $0.31(24)$ & $0.03(4)$ \\
\hline UV radiation & $0.65(48)$ & $0.54(92)$ \\
\hline
\end{tabular}


Cassini UVIS data suggest that the neutral source increased significantly $(>3 \mathrm{X})$ prior to the Cassini observing period. Assuming that the enhanced gas and dust both originate from volcanic plume activity, the neutral gas enhancement is consistent with the observed dust spike of Krüger et al. [2003]. In this case, it is interesting to note that a 3 order of magnitude dust enhancement only results in a $\sim 3 \mathrm{X}$ enhancement in the neutral source for the torus.

[24] A more detailed time-dependent model of the torus would not be appropriate without additional constraints. The specification of the hot electron component, for instance, adds a significant layer of complexity to the time dependent problem. One can obtain better fits to the data if the hot electron temperature is increased in proportion to the neutral source (as suggested by the steady state solution for 1 October). Thus one might be tempted to conclude that $T_{e h} \propto \mathcal{S}_{n}$ and/or $T_{e h} \propto 1 / \tau$ given that the hot electron components may be coupled to the plasma processes that drive transport, but such claims are well beyond the scope of this paper.

[25] The variations in the model neutral $\mathrm{O} / \mathrm{S}$ ratio between October $(\mathrm{O} / \mathrm{S}=1.7)$ and January $(\mathrm{O} / \mathrm{S}=1.9)$ should not be considered significant, as illustrated by our sensitivity study shown in Figure 5. However, it is worth considering how the torus would change if there were a change in atmospheric composition as a result of the enhanced neutral source. Russell et al. [2003] report temporal variations of Io's atmosphere based on ion cyclotron wave observations at Io. The Galileo spacecraft made several passes close to Io where ion cyclotron waves were found downstream from Io. Spatial day/night asymmetries in atmospheric composition predicted by Wong and Smyth [2000] (i.e., $\mathrm{SO}_{2}$ dominant on the dayside and SO dominant on the nightside) could not explain differences from pass to pass of the ion cyclotron power spectra; therefore Russell et al. [2003] concluded that these differences must reflect changes in the ion source, or atmospheric composition, due to variations in volcanic activity. Spencer et al. [2000] reports $\mathrm{S}_{2}$ in Io's Pele plume with $\mathrm{SO}_{2} / \mathrm{S}_{2}=3-12$ which could significantly alter the $\mathrm{O} / \mathrm{S}$ ratio if the composition of atmospheric escape is closely coupled to volcanic activity and plume composition. The possible activity of the Tvashtar volcano during this time may result in $\mathrm{S}_{2}$ enhancements similar to the Pele plume, though the results of our study are inconclusive with respect to compositional variations.

\section{Conclusions}

[26] 1. A significant change in the neutral source occurred near the beginning of October 2000, changing from $\geq 1.8$ tons $/ \mathrm{s}$ to $\sim 0.7$ tons $/ \mathrm{s}$.

[27] 2. The qualitative agreement between our model results and the Cassini observations suggest that an enhanced neutral gas production (e.g., $>3 \mathrm{X}$ ) is coupled with the observed $\sim 3$ order of magnitude increase in the Io dust emissions observed by Krüger et al. [2003].

[28] 3. Despite a substantial increase in the neutral production, only the $\mathrm{S}^{+}$and $\mathrm{S}^{+++}$mixing ratios vary significantly. Radiated power increases only $\sim 25 \%$ in response to the $\sim 3 \mathrm{X}$ increase in energy input as much of the excess energy is diverted to fast neutral escape due to ion-neutral charge exchange and increased transport rates.
[29] Acknowledgments. Peter Delamere and Fran Bagenal are supported by NASA grant NAG5-12994. Andrew Steffl's analysis of the Cassini UVIS data is supported under contract JPL 961196. The authors also thank Mihaly Horanyi and Harald Krueger for their helpful discussions regarding the iogenic dust emissions.

[30] Arthur Richmond thanks Frank Crary and another reviewer for their assistance in evaluating this paper.

\section{References}

Bagenal, F., F. J. Crary, A. I. F. Stewart, N. M. Schneider, D. A. Gurnett, W. S. Kurth, L. A. Frank, and W. R. Paterson (1997), Galileo measurements of plasma density in the Io torus, Geophys. Res. Lett., 24, 2119.

Barbosa, D. D. (1994), Neutral cloud theory of the Jovian nebula: Anomalous ionization effect of superthermal electrons, Astrophys. J., 430, 376.

Brown, M. E., and A. H. Bouchez (1997), The response of Jupiter's magnetosphere to an outburst on Io, Science, 278, 268.

Delamere, P. A., and F. Bagenal (2003), Modeling variability of plasma conditions in the Io torus, J. Geophys. Res., 108(A7), 1276, doi:10.1029/ 2002JA009706.

Dere, K. P., E. Landi, H. E. Mason, B. C. M. Fossi, and P. R. Young (1997), CHIANTI-An atomic dataset for emission lines, Astron. Astrophys. Suppl., 125, 149.

Esposito, L. W., J. E. Colwell, and W. E. McClintock (1998), Cassini UVIS observations of Saturns rings, Planet. Space Sci., 46, 1221.

Esposito, L. W., et al. (2004), The Cassini ultraviolet imaging spectrograph investigation, Space Sci. Rev., in press.

Krüger, H., P. Geissler, M. Horányi, A. L. Graps, S. Kempf, R. Srama, G. Moragas-Klostermeyer, R. Moissl, T. V. Johnson, and E. Grün (2003), Jovian dust streams: A monitor of Io's volcanic plume activity, Geophys. Res. Lett, 30(21), 2101, doi:10.1029/2003GL017827.

Lichtenberg, G., and N. Thomas (2001a), Detection of S (IV) $10.51 \mu \mathrm{m}$ emission from the Io plasma torus, J. Geophys. Res., 106, $29,899$.

McClintock, W. E., G. M. Lawrence, R. A. Kohnert, and L. W. Esposito (1993), Optical design of the Ultraviolet Imaging Spectrograph for the Cassini mission to Saturn, Opt. Eng., 32, 3038.

McGrath, M. A., P. D. Feldman, D. F. Strobel, H. W. Moos, and G. E. Ballester (1993), Detection of [oii] 2471 from the Io plasma torus, Astrophys. J., 415, 450

Meyer-Vernet, N., M. Moncuquet, and S. Hoang (1995), Temperature inversion in the Io plasma torus, Icarus, 116, 202.

Nelder, J. A., and R. Mead (1965), A simplex method for function minimization, Comput. J., 7, 308

Pontius, D. H., R. A. Wolf, T. W. Hill, R. W. Spiro, and Y. S. Yang (1998), Velocity shear impoundment of the Io plasma torus, J. Geophys. Res., $103,19,935$

Russell, C. T., X. Blanco-Cano, Y. L. Wang, and M. G. Kivelson (2003), Ion cyclotron waves at Io: Implications for the temporal variation of Io's atmosphere, Planet. Space Sci., 51, 937.

Saur, J., D. R. Strobel, F. M. Neubauer, and M. E. Summers (2003), The ion mass loading rate at Io, Icarus, 163,456

Schreier, R., A. Eviatar, and V. M. Vasyliunas (1998), A two-dimensional model of plasma transport and chemistry in the Jovian magnetosphere, J. Geophys. Res., 103, 19,901.

Shemansky, D. E. (1988), Energy branching in the Io plasma torus: The failure of neutral cloud theory, J. Geophys. Res., 93, 1773.

Smyth, W. H., and M. L. Marconi (2003), Nature of the iogenic plasma source in Jupiter's magnetosphere I. Circumplanetary distribution, Icarus, 166,85 .

Southwood, D. J., and M. G. Kivelson (1989), Magnetospheric interchange motions, J. Geophys. Res., 94, 299-308.

Spencer, J. R., K. L. Jessup, M. A. McGrath, G. E. Ballester, and R. V. Yelle (2000), Discovery of gaseous $S_{2}$ in Io's Pele plume, Science, 288, 1208.

Steffl, A., F. Bagenal, and A. I. F. Stewart (2004a), Cassini UVIS observations of the Io plasma torus: II. Radial variations, Icarus, in press.

Steffl, A., A. I. F. Stewart, and F. Bagenal (2004b), Cassini UVIS observations of the Io plasma torus: I. Initial results, Icarus, in press.

Thomas, N., F. Bagenal, T. W. Hill, and J. K. Wilson (2004), The Io neutral clouds and plasma torus, in Jupiter, Cambridge Univ. Press, New York, in press.

Wong, M. C., and W. H. Smyth (2000), Model calculations for Io's atmosphere at eastern and western elongations, Icarus, 146, 60-74.

Young, P. R., G. D. Zanna, E. Landi, K. P. Dere, H. E. Mason, and M. Landini (2003), CHIANTI: An atomic database for emission lines, part VI: Proton rates and other improvements, Astrophys. J., 144, 135.

F. Bagenal, P. A. Delamere, and A. Steffl, Laboratory for Atmospheric and Space Physics, CB 392 Duane Physics, University of Colorado, Boulder, CO 80309-0391, USA. (delamere@lasp.colorado.edu) 